\title{
Chord length distribution and the distance between two random points in a convex body in $\mathbb{R}^{n}$
}

\author{
Rafik Aramyan ${ }^{a, *}$, Daniel Yeranyan ${ }^{\mathrm{b}}$ \\ ${ }^{a}$ Russian-Armenian University, 123 Hovsep Emin Str., Yerevan 0051, Armenia. \\ ${ }^{b}$ Russian-Armenian University, 123 Hovsep Emin Str., Yerevan 0051, Armenia.
}

\begin{abstract}
In this article for $n$-dimensional convex body $\mathrm{D}$ the relation between the chord length distribution function and the distribution function of the distance between two random points in $\mathrm{D}$ was found. Also the relation between their moments was found.
\end{abstract}

Keywords: Integral geometry, Chord length distribution, Random points, Convex body. 2010 MSC: 53C65, 53C60, 31A10.

\section{Introduction}

The integral geometric concepts such as the distribution of the chord length, the distribution of the distance between two random points in a convex body $\mathrm{D}$ and many others carry some information about D. In this article the relation between the chord length distribution function and the distribution function of the distance between two random points uniformly distributed in a convex body was found. By $\mathbb{R}^{n}$ we denote the $n$-dimensional Euclidean space (here we assume that $n>2$, for case $n=2$ see [1]). Let $\mathrm{D} \subset \mathbb{R}^{n}$ be a compact convex set (convex body) with boundary $\partial \mathrm{D}$. By $V_{n}(\mathrm{D})$ we denote the $n-$ dimensional volume of $D$. By $S^{n-1}$ we denote the unit sphere in $\mathbb{R}^{n}$ centered at the origin $O$ and let $e_{O, \xi}$ be the hyperplane containing $\mathrm{O}$ and normal to $\xi \in S^{n-1}$. By $\mathbf{G}^{\mathfrak{n}}$ we denote the set of all lines in $\mathbb{R}^{\mathrm{n}}$. We use the usual parametrization of a line $\gamma=(\omega, P)$ : where $\omega \in S^{n-1}$ is the direction of $\gamma$ and $P$ is the intersection point of $\gamma$ and $e_{\mathrm{O}, \omega}$. By [D] we denote the set of lines intersecting $\mathrm{D}$. In $\mathrm{G}^{\mathrm{n}}$ we consider the invariant measure (with respect to the group of Euclidean motions) $\mu(\cdot)$. It is known that the element $d \gamma$ of the measure, up to a constant, has the following form ([2], [4], [6])

$$
\mathrm{d} \gamma=\mathrm{d} \omega \mathrm{dP},
$$

here $\mathrm{d} \omega$ and $\mathrm{dP}$ are elements of the Lebesgue measure on the sphere and the hyperplane, respectively. By $\chi(\gamma)$ we denote the length of a chord $D \cap \gamma$. We consider $\gamma$ as a random line intersecting $D$ with normalized measure $\frac{d \gamma}{\mu([D])}$. The distribution function $F_{\chi}(u)$ of $\chi(\gamma)$ is called the chord length distribution

\footnotetext{
*Corresponding author

Email addresses: rafikaramyan@yahoo.com (Rafik Aramyan), danielyeranyan@gmail.com (Daniel Yeranyan)
} 
function of D. Thus we have

$$
\mathrm{F}_{\chi}(\mathrm{u})=\frac{1}{\mu([D])} \int_{[\mathrm{D}]} \mathrm{I}_{\{\chi(\gamma)<\mathrm{u}\}} \mathrm{d} \gamma
$$

Now let $Q_{1}=\left(x_{1}, \ldots, x_{n}\right), Q_{2}=\left(y_{1}, \ldots, y_{n}\right)$ be random independent points uniformly distributed in $D$ and denote by $d$ distace between $Q_{1}$ and $Q_{2}$. By $F_{d}(t)$ we denote the distribution function of $d$. We have

$$
F_{d}(t)=\frac{1}{V_{n}(D)^{2}} \int_{\left\{\left(Q_{1}, Q_{2}\right):\left|Q_{1}-Q_{2}\right|<t, Q_{i} \in D, i=1,2\right\}} d Q_{1} d Q_{2}, \quad t \in \mathbf{R}^{1},
$$

here $d Q_{i}(i=1,2)$ is the usual Lebesgue's measure in $\mathbf{R}^{n}$.

\section{Main results}

Now we present the main results. The following theorem describes the relation between $F_{X}(t)$ and $F_{d}(t)$.

Theorem 2.1. Let $\mathrm{D}$ be a convex body. We have the following relation between $\mathrm{F}_{\mathrm{X}}(\mathrm{t})$ and $\mathrm{F}_{\mathrm{d}}(\mathrm{t})$

$$
\begin{gathered}
F_{d}(t)=\frac{V_{n-1}(\partial D) V_{n-2}\left(S^{n-2}\right)}{(n-1) V_{n}(D)^{2}}\left(-\frac{t^{n+1}}{n+1}+\frac{(n-1) V_{n}(D) V_{n-1}\left(S^{n-1}\right) t^{n}}{n V_{n-1}(\partial D) V_{n-2}\left(S^{n-2}\right)}+\right. \\
\left.\frac{t^{n}}{n} \int_{0}^{t} F_{X}(u) d u-\frac{1}{n} \int_{0}^{t} u^{n} F_{X}(u) d u\right) .
\end{gathered}
$$

Also in the article was found the following relation.

Theorem 2.2. Let $\mathrm{D}$ be a convex body. The following relation between the $\mathrm{k}$-th moment of the distance between two random points and the moments of the chord length distribution of $\mathrm{D}$ is valid

$$
E d^{k}=\frac{V_{n-1}(\partial D) V_{n-2}\left(S^{n-2}\right) E \chi^{n+k+1}}{(n-1)(n+k)(n+k+1) V_{n}(D)^{2}} .
$$

\section{Preliminary results}

In this section we need to prove the following lemmas.

Lemma 3.1. For the invariant measure of the lines intersecting $\mathrm{D}$ we have

$$
\mu([D])=\frac{V_{n-1}(\partial D) V_{n-2}\left(S^{n-2}\right)}{2(n-1)} .
$$

Proof of lemma 3.1. By definition we have

$$
\begin{gathered}
\mu([D])=\int_{[D]} d \gamma=\int_{[D]} d \omega d P=\frac{1}{2} \int_{S^{n-1}} d \omega \int_{D_{\omega}} d P= \\
\frac{1}{2} \int_{S^{n-1}} V_{n-1}\left(D_{\omega}\right) d \omega
\end{gathered}
$$


where $D_{\omega}$ is the orthogonal projection of $\mathrm{D}$ onto hyperplane $e_{\mathrm{O}, \omega}$. In this article, we consider a convex body $\mathrm{D}$ with positive Gaussian curvature at every point of $\partial \mathrm{D}$. For $\xi \in \mathrm{S}^{\mathrm{n}-1}$ we denote by $\mathrm{s}(\xi)$ the point on $\partial \mathrm{D}$ the outer normal of which is $\xi$. It is known that (see [3], [5])

$$
\mathrm{V}_{n-1}\left(\mathrm{D}_{\omega}\right)=\frac{1}{2} \int_{\partial \mathrm{D}}|\cos \widehat{(\omega, \xi)}| \mathrm{ds}(\xi),
$$

here $\mathrm{ds}(\xi)$ is the element of $n-1$-dimensional Lebesgue's measure on $\partial \mathrm{D}$. Substituting (3.3) into (3.2) and using the Fubini's theorem we obtain

$$
\begin{gathered}
\mu([D])=\frac{1}{4} \int_{S^{n-1}} \int_{\partial D}|\cos \widehat{(\omega, \xi)}| \mathrm{ds}(\xi) \mathrm{d} \omega= \\
\frac{1}{4} \int_{\partial \mathrm{D}} \int_{S^{n-1}}|\cos \widehat{(\omega, \xi)}| \mathrm{d} \omega \mathrm{ds}(\xi) .
\end{gathered}
$$

For any $\xi \in S^{n-1}$ we have (see [3])

$$
\int_{S^{n-1}}|\cos \widehat{(\omega, \xi)}| d \omega=\frac{2 V_{n-2}\left(S^{n-2}\right)}{n-1}
$$

thus

$$
\begin{aligned}
& \mu([D])=\frac{1}{4} \int_{\partial D} \int_{S^{n-1}}|\cos \widehat{(\omega, \xi)}| \mathrm{d} \omega \mathrm{d} s(\xi)=\frac{\mathrm{V}_{\mathrm{n}-2}\left(\mathrm{~S}^{\mathrm{n}-2}\right)}{2(\mathrm{n}-1)} \int_{\partial \mathrm{D}} \mathrm{d} s(\xi)= \\
& \frac{V_{n-1}(\partial D) V_{n-2}\left(S^{n-2}\right)}{2(n-1)}
\end{aligned}
$$

For a line $\gamma$ intersecting a convex body $\mathrm{D}$ we have the following lemma.

Lemma 3.2. Let $\chi(\gamma)$ be the length of the chord $\mathrm{D} \cap \gamma$. We have

$$
\int_{[D]} \chi(\gamma) d \gamma=\frac{V_{n}(D) V_{n-1}\left(S^{n-1}\right)}{2} .
$$

Proof of lemma 3.2. By definition we have $(\gamma=(\omega, \mathrm{P}))$

$$
\int_{[D]} \chi(\gamma) d \gamma=\frac{1}{2} \int_{S^{n-1}} d \omega \int_{D_{\omega}} \chi(\omega, P) d P .
$$

For any $\omega \in S^{n-1}$ it is obvious that $\chi(\omega, P) d P$ is the element of $n$-dimensional volume of $D$, hence the integrating by $d P$ over $D_{\omega}$ we get $V_{n}(D)$.

$$
\begin{gathered}
\int_{[D]} \chi(\gamma) d \gamma=\frac{1}{2} \int_{S^{n-1}} d \omega \int_{D_{\omega}} \chi(\omega, P) d P=\frac{V_{n}(D)}{2} \int_{S^{n-1}} d \omega= \\
\frac{V_{n}(D) V_{n-1}\left(S^{n-1}\right)}{2}
\end{gathered}
$$

It is known that a pair of points $Q_{1}, Q_{2}$ in $\mathbb{R}^{n}$ can be represented by the line $\gamma=(\omega, P)$ passing through the points and pair of one dimensional coordinates $\left(t_{1}, t_{2}\right)$ (see [6]). Thus

$$
\left(Q_{1}, Q_{2}\right)=\left(\gamma, t_{1}, t_{2}\right)=\left(\omega, P, t_{1}, t_{2}\right)
$$


Lemma 3.3. The Jacobian of the transform (3.10) is

$$
d Q_{1} d Q_{2}=\left|t_{1}-t_{2}\right|^{n-1} d t_{1} d t_{2} d \omega d P .
$$

Proof of lemma 3.3. For a fixed $\mathrm{Q}_{1}$ we represent $\mathrm{Q}_{2}$ by polar coordinates with respect to $\mathrm{Q}_{1}$. It is known that

$$
\mathrm{dQ}_{2}=\mathrm{r}^{\mathrm{n}-1} \mathrm{drd} \omega
$$

where $r=\left|Q_{1}-Q_{2}\right|$ and $\omega$ is the direction of $Q_{2}-Q_{1}$. For a fixed $\omega$ the point $Q_{1}$ can be represented by $P$ and $t_{1}$. Thus

$$
\mathrm{dQ}_{1}=\mathrm{dt}_{1} \mathrm{dP}
$$

and by multiplying (3.12) and (3.13) and taking into account that $r=\left|t_{1}-t_{2}\right|$ we get

$$
d Q_{1} d Q_{2}=\left|t_{1}-t_{2}\right|^{n-1} d t_{1} d t_{2} d \omega d P .
$$

\section{Proof of theorem 2.1}

By definition of the distribution function we have

$$
\mathrm{F}_{\mathrm{d}}(\mathrm{t})=\operatorname{Pr}\{\mathrm{d}<\mathrm{t}\}=\frac{1}{\mathrm{~V}_{\mathrm{n}}(\mathrm{D})^{2}} \int_{\mathrm{d}<\mathrm{t}} \mathrm{dQ}_{1} \mathrm{dQ}_{2}
$$

Using (3.11) from lemma 3.3 we get

$$
F_{d}(t)=\frac{1}{V_{n}(D)^{2}} \int_{[D]} \int_{\left|t_{1}-t_{2}\right|<t}\left|t_{1}-t_{2}\right|^{n-1} d t_{1} d t_{2} d \gamma .
$$

We represent the integral by the form

$$
\int_{[D]} \int_{\left|t_{1}-t_{2}\right|<t}\left|t_{1}-t_{2}\right|^{n-1} d t_{1} d t_{2} d \gamma=\int_{[D]} I_{\{\chi(\gamma) \geqslant t\}} J_{1}(t)+I_{\{\chi(\gamma)<t\}} J_{1}(t) d \gamma
$$

where

$$
J_{1}(t)=\int_{\left|t_{1}-t_{2}\right|<t}\left|t_{1}-t_{2}\right|^{n-1} d t_{1} d t_{2} .
$$

After calculating $\mathrm{J}_{1}$ we obtain that

$$
J_{1}(t)=\frac{-2 t^{n+1}}{n+1}+\frac{2 t^{n}}{n} x(\gamma)
$$

for $\chi(\gamma) \geqslant t$ and

$$
J_{1}(t)=\frac{2(\chi(\gamma))^{n+1}}{n(n+1)}
$$

for $\chi(\gamma)<\mathrm{t}$.

$$
\begin{gathered}
F_{d}(t)=\frac{1}{V_{n}(D)^{2}} \int_{[D]}\left(I_{\{\chi(\gamma) \geqslant t\}}\left(\frac{-2 t^{n+1}}{n+1}+\frac{2 t^{n}}{n} \chi(\gamma)\right)+\right. \\
\left.I_{\{\chi(\gamma)<t\}}\left(\frac{2(\chi(\gamma))^{n+1}}{n(n+1)}\right)\right) d \gamma .
\end{gathered}
$$


Now we denote by $\mathrm{J}_{2}, \mathrm{~J}_{3}, \mathrm{~J}_{4}$ the following integrals

$$
\begin{gathered}
J_{2}(t)=\int_{[D]} I_{\{\chi(\gamma) \geqslant t\}} d \gamma, \\
J_{3}(t)=\int_{[D]} I_{\{\chi(\gamma) \geqslant t\}} \chi(\gamma) d \gamma, \\
J_{4}(t)=\int_{[D]} I_{\{\chi(\gamma)<t\}}(\chi(\gamma))^{n+1} d \gamma .
\end{gathered}
$$

Easy to notice (using (3.1) from lemma 3.1) that

$$
J_{2}(t)=\frac{V_{n-1}(\partial D) V_{n-2}\left(S^{n-2}\right)}{2(n-1)}\left(1-F_{x}(t)\right)
$$

To calculate $\mathrm{J}_{3}$ we change it the following way

$$
\begin{gathered}
\mathrm{J}_{3}(\mathrm{t})=\int_{[D]} \mathrm{I}_{\{\chi(\gamma) \geqslant t\}} \chi(\gamma) \mathrm{d} \gamma=\int_{[D]}\left(1-\mathrm{I}_{\{\chi(\gamma)<\mathrm{t}\}}\right) \chi(\gamma) \mathrm{d} \gamma= \\
\frac{\mathrm{V}_{\mathrm{n}}(\mathrm{D}) \mathrm{V}_{\mathrm{n}-1}\left(\mathrm{~S}^{n-1}\right)}{2}-\int_{[D]} \mathrm{I}_{\{\chi(\gamma)<\mathrm{t}\}} \chi(\gamma) \mathrm{d} \gamma
\end{gathered}
$$

(see (3.7) from lemma 3.2) then denote

$$
\mathrm{J}_{5}(\mathrm{t})=\int_{[\mathrm{D}]} \mathrm{I}_{\{\chi(\gamma)<\mathrm{t}\}} \chi(\gamma) \mathrm{d} \gamma
$$

and calculate it instead. First we calculate the derivative of $J_{5}$ and then intergrate from 0 to $t\left(J_{5}(0)=0\right)$. Using the first mean value theorem for definite integrals we have

$$
\begin{gathered}
\frac{d}{d t} J_{5}(t)=\lim _{\Delta t \rightarrow 0} \frac{J_{5}(t+\Delta t)-J_{5}(t)}{\Delta t}=\lim _{\Delta t \rightarrow 0} \frac{1}{\Delta t} \int_{[D]} I_{\{t \leqslant \chi(\gamma)<t+\Delta t\} \chi}(\gamma) d \gamma= \\
\lim _{\Delta t \rightarrow 0} \frac{V_{n-1}(\partial D) V_{n-2}\left(S^{n-2}\right) t\left(F_{X}(t+\Delta t)-F_{X}(t)\right)}{2(n-1) \Delta t}= \\
\frac{V_{n-1}(\partial D) V_{n-2}\left(S^{n-2}\right)}{2(n-1)} t_{\chi}(t)
\end{gathered}
$$

and after integrating that we get

$$
J_{5}(t)=\frac{V_{n-1}(\partial D) V_{n-2}\left(S^{n-2}\right)}{2(n-1)}\left(t F_{x}(t)-\int_{0}^{t} F_{x}(u) d u\right) .
$$

For $\mathrm{J}_{3}$ we have

$$
\begin{gathered}
J_{3}(t)=\frac{V_{n}(D) V_{n-1}\left(S^{n-1}\right)}{2}- \\
\frac{V_{n-1}(\partial D) V_{n-2}\left(S^{n-2}\right)}{2(n-1)}\left(t F_{x}(t)-\int_{0}^{t} F_{x}(u) d u\right) .
\end{gathered}
$$

Using the same technique we can calculate $\mathrm{J}_{4}$ and get that

$$
J_{4}(t)=\frac{V_{n-1}(\partial D) V_{n-2}\left(S^{n-2}\right)}{2(n-1)}\left(t^{n+1} F_{x}(t)-(n+1) \int_{0}^{t} u^{n} F_{\chi}(u) d u\right) .
$$


After substituting (4.11), (4.16), (4.17) into (4.7) finally we obtain that

$$
\begin{gathered}
F_{d}(t)=\frac{V_{n-1}(\partial D) V_{n-2}\left(S^{n-2}\right)}{(n-1) V_{n}(D)^{2}}\left(-\frac{t^{n+1}}{n+1}+\frac{(n-1) V_{n}(D) V_{n-1}\left(S^{n-1}\right) t^{n}}{n V_{n-1}(\partial D) V_{n-2}\left(S^{n-2}\right)}+\right. \\
\left.\frac{t^{n}}{n} \int_{0}^{t} F_{x}(u) d u-\frac{1}{n} \int_{0}^{t} u^{n} F_{x}(u) d u\right)
\end{gathered}
$$

Differentiating the distribution function $F_{d}(t)$ we get the following expression for the density function $f_{d}(t)$ of $d$

$$
\begin{gathered}
f_{d}(t)=\frac{V_{n-1}(\partial D) V_{n-2}\left(S^{n-2}\right)}{(n-1) V_{n}(D)^{2}}\left(-t^{n}+\frac{(n-1) V_{n}(D) V_{n-1}\left(S^{n-1}\right) t^{n-1}}{V_{n-1}(\partial D) V_{n-2}\left(S^{n-2}\right)}+\right. \\
\left.t^{n-1} \int_{0}^{t} F_{X}(u) d u\right) .
\end{gathered}
$$

Note that in $\mathbb{R}^{2}$ formula (4.19) first was found in [1]. Now we are going to prove theorem 2.2.

\section{Proof of theorem 2.2}

We can prove that theorem by just putting the (4.19) in moments formula

$$
E d^{k}=\int_{-\infty}^{\infty} t^{k} f_{d}(t) d t
$$

but we will do that by the following way

$$
\begin{aligned}
& E d^{k}=\frac{1}{V_{n}(D)^{2}} \int_{Q_{1}, Q_{2} \in D}\left|Q_{1}-Q_{2}\right|^{k} d_{Q_{1}} d Q_{2}= \\
& \frac{1}{V_{n}(D)^{2}} \int_{[D]} \int_{0}^{x(\gamma)} \int_{0}^{x(\gamma)}\left|t_{1}-t_{2}\right|^{n+k-1} d t_{1} d t_{2} d \gamma= \\
& \frac{2}{V_{n}(D)^{2}(n+k)(n+k+1)} \int_{[D]}(x(\gamma))^{n+k+1} d \gamma= \\
& \frac{V_{n-1}(\partial D) V_{n-2}\left(S^{n-2}\right) E \chi^{n+k+1}}{(n-1)(n+k)(n+k+1) V_{n}(D)^{2}}
\end{aligned}
$$

\section{References}

[1] N. G. Aharonyan, The Distribution of the Distance Between Two Random Points in a Convex Set, Russian Journal of Mathematical Research. Series A, 2015, Vol.(1), pp. 1-5. 1, 4

[2] Ambartzumian, R. V., Combinatorial integral geometry, metric and zonoids. Acta Appl. Math. 1987, vol. 9, 3 - 27. 1

[3] R.H. Aramyan, "The Sine Representation of Centrally Symmetric Convex Bodies", Journal of Contemporary Mathematical Analysis (Armenian Academy of Sciences), 2018, Vol. 53, No. 6, pp. 307- 312. 3, 3

[4] R Aramyan, A Flag representation for n-dimensional convex body, The Journal of Geometric Analysis, vol. 29 (3), 2019, pp. 2998-3009. https: / / doi.org/10.1007/s12220-018-00102-1 1

[5] W. Blaschke, Kreis und Kugel, (in German), 2nd Ed., W. de Gruyter, Berlin, 1956. 3

[6] L.A. Santalo, Integral Geometry and Geometric Probability, Addison-Wesley, Reading, Massachusetts, USA (1976). 1,3 